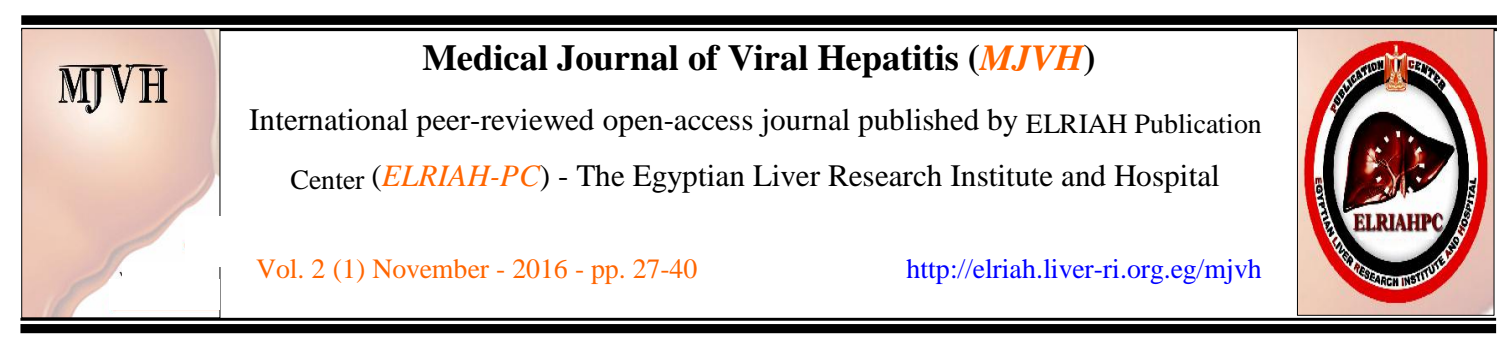

Original article

\title{
Fibrogenic/Angiogenic Linker for Non-invasive assessment of hepatic fibrosis staging in chronic hepatitis $\mathrm{C}$ genotype 4 patients
}

\author{
Toson, E. ${ }^{1}$, Shiha, G. ${ }^{2,3}$, Abdelgaleel A. ${ }^{4}$ \\ ${ }^{1}$ Biochemistry Division, Chemistry Dept. Faculty of Science, Damietta University, Damietta, Egypt. \\ ${ }^{2}$ Internal Medicine Department, Faculty of Medicine, Mansoura University, Mansoura, Egypt. \\ ${ }^{3}$ Egyptian Liver Research Institute and Hospital (ELRIAH), Sherbin, El-Mansoura, Egypt. \\ ${ }^{4}$ Chemistry Department, Faculty of Science, Damietta University, Damietta, Egypt.
}

* E-mail: eatoson@yahoo.com

Article History

Received: 16/6/2016

Revised: $23 / 10 / 2016$

Accepted: 25/10/2016

\section{Keywords:}

Hyaluronic acid

Hyaluronic acid vascular

\section{score}

Vascular endothelial growth

factor,

Liver fibrosis

Hepatitis C virus

Genotype 4

\begin{abstract}
:
Liver biopsy is the golden standard but has its complications. Our aim is to establish a method to assess hepatic fibrosis both directly and indirectly, correctly and non-invasively in chronic hepatitis $C$ genotype 4 patients. Also, the diagnostic power of this method will be compared with those of APRI, AAR, FCI, FI, LOK, FIB4, GUCI and KING scores. Samples were collected from 220 patients (F0-F4) of whom 100 were used in the validation study. Hyaluronic acid (HA) and vascular endothelial growth factor (VEGF) levels, HCV RNA, liver function tests, platelet counts, and liver biopsy were done. HA and VEGF levels were correlated with the severity of the disease and acting as fibrogenic/angiogenic cross linker. The areas under receiver operating characteristic curve (AUCs) of the developed method were 0.979 and 0.994 for significant (F2-F4) and advanced fibrosis (F3-F4) (cut off= 0.583 and 6.27, respectively). These AUCs were directly based on HA and VEGF and indirectly on AAR and is termed HA vascular (HAV) method $=-35.1+0.14(H A)(n g / L)+0.03(V E G F)(\mathrm{pg} / \mathrm{ml})+(-6.7)$ (AAR). Surprisingly, the validation study of this cross linker gave numerical values of AUCs near unity i.e. 0.990, 0.996 and 0.995 for significant, advanced and liver cirrhosis. Also, the indirect published scores gave lower AUCs compared with those of the developed one. Our developed method can not only help to assess liver fibrosis staging effectively but also avoid the invasiveness and the limitations of liver biopsy in such patients.
\end{abstract}

\section{Introduction}

The liver is a vital organ in the body because it performs many important functions e.g. detoxification, protein synthesis and help in digestion [1]. It is prone to many diseases including viral 
hepatitis $\mathrm{C}$ virus (HCV) which cause its inflammation [2] with subsequent acute or chronic disorders. These disorders may lead to liver fibrosis, cirrhosis, hepatocellular carcinoma (HCC), and finally death [3]. Globally, HCV is considered a major health problem. This is because; more than 180 million individuals are chronically infected with HCV [4]. Virological diagnosis of $\mathrm{HCV}$ infection is based on two categories of laboratory tests; detecting specific antibody to HCV (anti-HCV) (indirect tests) and assays that can detect, quantify, or characterize the components of $\mathrm{HCV}$ viral particles, such as HCV RNA and core antigen (direct tests) [5]. Liver fibrosis is the scarring process that is produced from the excessive accumulation of extracellular matrix (ECM) proteins [6,7] that form fibrous scar tissue in the liver. The formation of the latter distorts the organization of hepatic architectures and develops nodules of renewal hepatocytes, disrupts blood flow through the liver and finally produces hepatocellular dysfunctions [6].Liver biopsy, which is staged by Metavair (stages 0-4) and Ishak score (stages 0-5) [8, 9], is still the golden standard for assessment of hepatic fibrosis although its invasiveness, sampling errors and complications [10]. Nowadays, there is a need for reliable, simple, and noninvasive methods for staging liver fibrosis. These methods contain routine laboratory tests, such as serum aminotransferase, platelet count, serum albumin level and prothrombin time (indirect markers) [11,12]. In addition, serum levels of proteins, which are directly related to hepatic fibrogenesis, are used as surrogate markers of such process. These include collagen type III, HA, tissue inhibitor of metalloproteinase type 1 (TIMP-1) and vascular endothelial growth factor VEGF [13]. Serum HA level increases with the development of fibrosis and/or cirrhosis [14]. Generally, the imbalance between ECM degradation and production lead to liver fibrogenesis [15]. Liver sinusoidal endothelium (LSE) plays an important role in stabilizing tissues and in the organization of the growth and differentiation of cells. Cirrhotic livers are characterized by transformation of the LSE into a continuous, vascular type, i.e. sinusoidal endothelial cells (SECs) capillarization. HA is a serum marker which can effectively reflect such capillarization process. The latter process; initiates response from hepatic stellate cell (HSC) that gets activated by oxidative stress resulting in the production of larger amounts of ECM components including HA which exegrravated SECs capillarization. Under these conditions, if the liver regeneration fails, the hepatocytes are substituted with abundant fibrillar collagen [16]. Such transformations lead to hypoxia [17] through mechanisms requiring the participation of proinflammatory cytokines and growth factors [18]; including vascular endothelial growth factor (VEGF). The latter promote and regulate endothelial cell proliferation and migration, matrix remodeling, recuritment of pericytes and neovessels stabilization in a process called angiogenesis [18]. This is because it acts as a highly specific mitogen for endothelial cells by increasing vascular permeability through disorganization of endothelial junctional proteins [19,20]. Angiogenesis is an essential process for organ growth and repair during and after hepatic diseases [21].The disruption of the balance between hepatic fibrogenesis and angiogenesis can lead to several diseases including malignancy [22]. Therefore, the aim of this study is to develop and validate a predictive method for staging liver fibrosis to avoid complications of 
liver biopsy. Also, its diagnostic power was compared with those of eight published non-invasive scores; namely, APRI, AAR, FCI, FI, LOK, FIB4, GUCI and KING.

\section{Patients and Methods 2.1. Patients}

This study was conducted on 220 patients (F0-F4) of whom 100 were used in the validation study with hepatitis $\mathrm{C}$ virus which were selected from The Egyptian Liver Institute and Hospital (ELRIAH) in Dakahlia, Egypt. The patients were randomly chosen from adult males and females. All patients were negative for other causes of chronic liver diseases and having normal kidney function, normal glucose and with no liver transplantation. All patients were tested positive for the presence of HCV RNA using quantitative polymerase chain reaction (PCR) and HCVAbs using Enzyme linked immunosorbent assay (ELISA). The study was approved by the ethical committee of Mansoura Medical School and informed consent were taken from all participants.

\subsection{Samples and blood markers}

\subsubsection{Blood samples}

They were withdrawn by vein puncture from all cases and were divided into two portions. The first was collected on EDTA-K3 (EDM, Cairo, Egypt) for determination of platelet count. Serum was separated from the second portion and was used for assessment of HA, VEGF, HCV RNA and HCVAbs. The latter were kept frozen at $-80^{\circ} \mathrm{C}$ until use in each case.

\subsubsection{Blood markers}

\subsubsection{Biochemical investigations}

The following were measured in the serum of each patient and control subject.

- Human Hyaluronic Acid (HA) and Vascular Endothelial Growth Factor (VEGF)
HA and VEGF used were bought from WKEA MED SUPPLIES CORP (206 building 6, Chenguang Gardon, Qianjin street; Changchan 130012 China). The reaction based on sandwich ELISA technique according to the enclosed pamphlets [19].

- Routine liver function tests and blood picture

They include; albumin (Alb), Alanine amino-transferase (ALT) and Aspartate amino- transferase (AST), total bilirubin and alkaline phosphatase (ALP) using automated Biochemistry analyzer.

\subsubsection{Haematological parameters}

Complete blood pictures including Platelets were performed on D-cell 60 automated Hematology analyzer (D-cell 60; Diagon Ltd, Budapest, Hungary). Prothrombin activity was done and INR was calculated.

\subsubsection{Serological and molecular markers}

Serological markers for detecting $\mathrm{HCV}$ antibodies were done using ELISA kit which was provided by Merieux anti$\mathrm{HCV}$, version 4.0, Diasorin S.P.A. via Crescent no 13040 Saluggia (VC) (Italy). Molecular detection of HCV RNA was done by quantitative PCR using QIAamp viral RNA extraction kit (Qiagen USA cat \#52906).

\subsubsection{Histopathological examination}

Liver biopsies were performed and stained with hematoxyline- eosin as well as Masson tri-chrome stains [17]. METAVIR scoring system was done: F0 (no fibrosis), F1 (mild fibrosis without septa), F2 (moderate fibrosis with few septa), F3 (severe fibrosis with numerous septa but without cirrhosis) and F4 (cirrhosis). After staging of liver fibrosis, the patients were classified using two classifications; the first includes F0-F1 (non-significant fibrosis) and F2, F3 and F4 (significant fibrosis), the second 
includes F0, F1, F2 (non-severe fibrosis) and F3, F4 (severe fibrosis) [23].

\subsection{Formulas of the selected Scores}

1- AST platelets ratio index (APRI) was calculated using Wais formula [23] (AST (upper limit of normal)/ALT (IU/L) $\times 100$ )/ platelet count (platelets x $10^{9} / \mathrm{L}$ ) $\mathrm{X} 100$ 2- FIB4 index was calculated using Sterling's formula [24] Age (years) x AST (IU/L)/platelet count $\left(10^{9} / \mathrm{L}\right)$ x ALT (IU/L)1/2 3- Göteborg University Cirrhosis Index (GUCI) using this formula [25] (ASTxINRx100)/platelet count $\left(10^{9} / \mathrm{L}\right)$ 4- King's score using this formula [26] Age (years) x AST (IU/L) x INR/platelet count $\left(10^{9} / \mathrm{L}\right) 5$-LOK (Model 3) using this formula [26] Log odds $=-5.56$ - $0.0089 \times$ platelet $\left(\times 10^{9} / \mathrm{L}\right)+1.26 \times$ AST/ ALT ratio + 5.27 $\times$ INR. 6Fibrosis index (FI) was calculated using this formula [27] as: $8.0-0.01 \mathrm{x}$ platelet count $\left(\times 10^{9} / \mathrm{L}\right)$ - serum albumin $(\mathrm{g} / \mathrm{dl}) 7$ AAR: AST/alanine aminotransferase (ALT) ratio (AAR) [27]. 8- Fibrosis-cirrhosis index (FCI): [(ALP $\times$ Bilirubin) / (Albumin $\times$ Platelet count)] [27].

\subsection{Study Approval and patients, consent}

All patients were informed about the study details then signed a written informed consent. With respect to patients' confidentiality, patients were represented in the study by code numbers. All personal data were included. The study protocol conf-ormed to the ethical guidelines of the 1975 Declaration of Helsinki as reflected in a priori approval by the institution's human research committee.

\subsection{Statistical analysis}

Statistical analyses were performed by Medcalc software version 15 (Medcalc 15, Mariakerke, Belgium). Continuous variables were expressed as mean \pm standard error of mean (SEM). Comparisons of fibrogenic and angiogenic markers as well as routine laboratory tests and fibrosis stages were analyzed by MannWhitney U-test using a two-sided Pvalue. The main endpoint was the identification of patients with clinically significant fibrosis (F2-F4) versus those without (F0-F1) using a simple combination of routine laboratory markers. The cutoff values for optimal clinical performance measure were determined from ROC curves. The cut-offs selected from the ROC curve were those that best identified significant fibrosis (F2-F4), advanced fibrosis (F3-F4). A value of $P$ $<0.05$ was considered statically significant. ROC curve was done to determine the cutoff point, AUC, sensitivity, specificity, PPV and NPV of presences of fibrosis to define diagnostic accuracy.

\section{Results}

\subsection{Patients' data}

According to Metavair score, 20 $(16.5 \%)$ patients were in $\mathrm{F} 0$ and $\mathrm{F} 1$ in 46 (38\%), F2 in 26 (22\%), F3 in 20 (16.5\%) and $8(7 \%)$ patients in F4, tab. (1).

\subsection{Diagnosis performance of can- didate markers}

Liver biochemical tests (serum albumin, ALT, AST, total bilirubin and alkaline phosphatase) were significantly different in various groups in both classifications, tab. (2). The activities of both ALT and AST and the level of HA and VEGF were increased in sera of patients with significant fibrosis as well as in those with severe fibrosis compared with those of non-significant and nonsevere fibrosis, respectively, while ALP, albumin and platelet count were decreased in the blood of patients with significant fibrosis. Comparing the ability of the latter markers to differentiate between groups of both classifications, it was found that the extent of increase of HA, VEGF, ALT and AST in severe fibrosis $(\mathrm{F} 3-\mathrm{F} 4, \mathrm{n}=14)$ was higher than that of the significant fibrosis (F2-F4, $n=27)$. In each case, the AUCs were used for identification of patients with significant fibrosis from those with non-significant fibrosis. The AUCs were 0.941 and 0.911 for HA and VEGF, respectively. 
The basic diagnostic power of HA has specificity (94\%) and sensitivity (85\%) PPV $(92 \%)$ and NPV $(89 \%)(\mathrm{p}<0.0001)$. In addition, those of VEGF the specificity was $(91 \%)$, sensitivity (78\%), PPV of $88 \%$ and NPV of $83 \%(\mathrm{p}<0.0001)$. Also, the levels of HA and VEGF can differentiate patients with severe from those with a non-severe fibrosis. AUC of HA is 0.959 with specificity of 89 , sensitivity of 93 , PPV of $72 \%$, NPV of $98 \%$, ( $p<0.0001$ ), fig. (1-a) and AUC of VEGF is 0.994 with specificity (93\%), sensitivity (100\%), PPV of $82 \%$ and NPV of $100 \%$ $(p<0.0001)$, tab. (2), fig. (1b). As we expected, the performance characteristics after combining the individual values of HA or VEGF with the numerical values of the previously published 8 noninvasive scores at their optimal Cut off for the discrimination between significant and non-significant liver fibrosis were enhanced. In addition, the same combination enhanced the discrimination between severe and non-severe liver fibrosis, tab. (3). Therefore, we tested whether the combination between HA and VEGF only would help in discriminating the liver fibrosis stages or not. For this reason the individual results of both HA and VEGF were tested for the latter discriminating activity using logistic regression. The equation was found to be: $\mathrm{HA}+\mathrm{VEGF}=-34.119+$ 0.1126 (HA) (ng/L) + 0.0245 (VEGF) (pg/ml). By applying such equation on the estimation study, the AUROC of such combination was 0.897 in differentiating patients with significant from non significant fibrosis and was 0.996 in differentiating patients with severe from non severe fibrosis $(p<0.0001)$, tab. (4), fig. (2-a,b).

\subsection{Development of HA-Vascular score (HAV score)}

Using logistic regression analyses that combine the biomarkers evaluated in our patients to create several predictions for staging liver fibrosis in patients with non-significant fibrosis (F0-F1), the best linear combination of blood markers that were selected by the multivariate discriminate analysis for the development of a novel simple noninvasive score was called HA-Vascular score (HAV score). The score was initially based on HA and VEGF and then on AAR, The first two were direct markers of hepatic fibrosis and the third one was indirect. The HAV score was able to differentiate non-significant fibrosis from significant one with an AUC of 0.979 and at a cut off $>0.583$ $(\mathrm{p}<0.0001)$, fig. (3-a), Also, it can differentiate patients with non-severe fibrosis from the severe one with AUC of 0.994 at a cut off $>6.27(\mathrm{p}<0.0001)$, tab. (4), fig. (3-b). HAV score $=-35.1+0.14(\mathrm{HA})$ $(\mathrm{ng} / \mathrm{L})+0.03$ (VEGF) $(\mathrm{pg} / \mathrm{ml})+(-6.7)$ (AAR) (IU/L).

\subsection{Validation study}

The validation group included 100 HCV patients with matched clinical and pathological investigations as well as the inclusion and exclusion criteria as those for the estimation study. There was no significant difference between the values of the laboratory blood tests in the estimation and in the validation studies. Our validation study only contains the best 5 non invasive scores (AAR, GUCI, FI, KING and APRI). Table (5) illustrates the diagnostic accuracies of HAV score for discriminating significant fibrosis, advanced fibrosis and cirrhosis patients. The HAV score was able to differentiate patients with significant fibrosis (F2-F4) at a cut-off of $>0.583$ in the validation group with an AUC of 0.990 , PPV of $100 \%$, NPV of $93 \%$, specificity of 100 , sensitivity of 92. Also, HAV score produced an AUC of 0.996 with PPV of $93 \%$, NPV of $100 \%$, specificity of $97 \%$, sensitivity of $100 \%$ at cutoff point $>-4.4$ $(p<0.0001)$ for identify advanced fibrosis. In addition, in cirrhotics; HAV score produced an AUC of 0.995 with PPV of $80 \%$, NPV of $100 \%$, specificity of $98 \%$, sensitivity of $100 \%$ and at a cutoff point $>19.5(\mathrm{P}<0.0001)$, tab. (5). Surprisingly, there was no significant difference between the diagnostic performance of HAV in the estimation and in the validation study. 
Table (1) Baseline characteristics of chronic hepatitis C patients with hepatic fibrosis regard two main classifications of hepatic fibrosis

\begin{tabular}{|c|c|c|c|c|c|c|c|}
\hline-- &.- & \multicolumn{3}{|c|}{$\mathrm{l}^{\text {st }}$ fibrotic classification } & \multicolumn{3}{|c|}{$2^{\text {nd }}$ fih rotic c lassific a tion } \\
\hline-- & Normal & $\begin{array}{c}\text { Non- } \\
\text { significant } \\
(F 0,1, n=66, \\
F 0=20 \\
F 1=46)\end{array}$ & $\begin{array}{c}\text { Significant } \\
(F 2,3,4, n=54, \\
F 2=26, F 3=20, F \\
4=8)\end{array}$ & P-value & $\begin{array}{c}\text { Non-severe } \\
(F 0,1,2, n=9 \\
0)\end{array}$ & $\begin{array}{c}\text { Severe } \\
(F, 3,4, n= \\
28)\end{array}$ & P-value \\
\hline Age (year) & $30.6 \pm 7.5$ & $36.5 \pm 10.3$ & $51.9 \pm 11.2$ & $<0.0001$ & $39 \pm 10.8$ & $57.9 \pm 9.4$ & $<0.0001$ \\
\hline ALT (IU/L) & $21.1 \pm 5.6$ & $28.8 \pm 14.4$ & $50.0 \pm 25.8$ & $<0.0001$ & $30.8 \pm 14.2$ & $63.3 \pm 28$ & $<0.0001$ \\
\hline AST (IU/L) & $18.6 \pm 4.5$ & $26.5 \pm 10.6$ & $35.4 \pm 24.5$ & 0.0692 & $26.6 \pm 9.7$ & $43.3 \pm 31.9$ & 0.0261 \\
\hline ALP (IU/L) & $74.5 \pm 19.1$ & $73.7 \pm 25.1$ & $68.1 \pm 23.9$ & 0.2985 & $73.3 \pm 23.7$ & $64.5 \pm 26.8$ & 0.2763 \\
\hline$A \mathrm{lb}(\mathrm{g} / \mathrm{dl})$ & $4.7 \pm 0.18$ & $4.5 \pm 0.26$ & $4.38 \pm 0.22$ & 0.0007 & $4.6 \pm 0.3$ & $4.3 \pm 0.13$ & $<0.0001$ \\
\hline Bili. T (mg/dl) & $0.65 \pm 0.15$ & $0.5 \pm 0.14$ & $0.7 \pm 0.2$ & 0.7082 & $0.6 \pm 0.15$ & $0.7 \pm 0.2$ & 0.5188 \\
\hline Platelet $\left(\mathrm{l}^{6} / \mathrm{L}\right)$ & $273.2 \pm 47$ & $252.2 \pm 62$ & $218.6 \pm 52$ & 0.0181 & $246.3 \pm 60$ & $206.9 \pm 51$ & 0.0133 \\
\hline $\mathrm{HA}$ (ng/L) & $52.4 \pm 15.0$ & $225.3 \pm 18$ & $\begin{array}{c}304.2 \pm 64.3 \\
{[135 \%] \dagger}\end{array}$ & $<0.0001$ & $236.5 \pm 27$ & $\begin{array}{c}340.6 \pm 67.0 \\
{[143.5 \%] \ddagger}\end{array}$ & $<0.0001$ \\
\hline VEGF (pg/ml) & $108.6 \pm 12.2$ & $186 \pm 48$ & $\begin{array}{c}308.5 \pm 80 \\
{[166 \%] \dagger}\end{array}$ & $<0.0001$ & $203 \pm 57$ & $\begin{array}{c}364 \pm 60.5 \\
{[178 \%] \ddagger}\end{array}$ & $<0.0001$ \\
\hline
\end{tabular}

ALT: Alanine amino-transferase, AST: Aspartate aminotransferase, Alb: Albumin, ALP: Alkaline phosphatase (IU/L), HA: Hyaluronic acid, VEGF: Vascular Endothelial Growth Factor and SD: standard deviations, values were expressed as mean $\pm S D$, ( ): values between () represent the \% of change, [ ]\%: Percent of change compared to Non- significant fibrosis, .[ ]\%: Percent of change compared to Non- severe.

Table (2) Comparison of the diagnostic values of hyaluronic acid (HA), vascular endothelial growth factor (VEGF) and those of AAR, GUCI, FCI, FI, LOK, FIB4, KING and APRI at their original cut-off in staging of liver fibrosis.

\begin{tabular}{|c|c|c|c|c|c|c|c|c|c|c|c|c|c|c|}
\hline Group & \multicolumn{7}{|c|}{ Non-significant 7 Significant } & \multicolumn{7}{|c|}{ Non-severe Z Severe } \\
\hline Parameter & Cut off & $A U C$ & $S p$ & $S n^{*}$ & $P P V$ & $\mathrm{NPV}$ & $p$ & Cut off & $A U C$ & Sp & $S n^{*}$ & $P P V$ & $\mathrm{NPV}$ & $p$ \\
\hline $\mathrm{HA}(\mathrm{ng} / \mathrm{L})$ & $>252$ & 0.941 & 94 & 85 & 92 & 89 & $\begin{array}{c}0.000 \\
1\end{array}$ & $>271$ & 0.959 & 89 & 93 & 72 & 98 & $\begin{array}{c}0.000 \\
1\end{array}$ \\
\hline VE GF (pg/ml) & 255 & 0.911 & 91 & 78 & 88 & 83 & $\begin{array}{c}<0.000 \\
1\end{array}$ & $>289$ & 0.994 & 93 & 100 & 82 & 100 & $\begin{array}{c}<0.000 \\
1\end{array}$ \\
\hline$A A R$ & $? 0.91$ & 0.700 & 94 & 48 & 87 & 68 & 0.0065 & $>1.0$ & 0.748 & 76 & 71 & 48 & 90 & 0.0021 \\
\hline GUCI & $>1.0$ & 0.672 & 79 & 56 & 68 & 68 & 0.0146 & $>1.56$ & 0.736 & 83 & 64 & 53 & 88 & 0.0056 \\
\hline $\mathrm{FCI}$ & $>0.095$ & 0.539 & 33 & 81 & 50 & 69 & 0.6103 & $>1.25$ & 0.540 & 52 & 71 & 31 & 86 & 0.6772 \\
\hline FI & $>3.3$ & 0.724 & 94 & 44 & 86 & 67 & 0.0008 & $>3.56$ & 0.783 & 89 & 64 & 64 & 89 & 0.0002 \\
\hline $\mathrm{LOK}$ & $>0.5$ & 0.556 & 58 & 59 & 53 & 63 & 0.4675 & $>0.76$ & 0.618 & $6 ?$ & 64 & 38 & 86 & 0.2230 \\
\hline FIB4 & $>2.6 ?$ & 0.600 & 58 & $6 ?$ & 56 & 68 & 0.1762 & $\$ 3.25$ & 0.606 & 85 & 43 & 46 & 83 & 0.2348 \\
\hline KING & $>17.5$ & 0.802 & 88 & 63 & 81 & 74 & $<0.0001$ & $\$ 17.3$ & 0.859 & 74 & 86 & 50 & 94 & $<0.0001$ \\
\hline APRI & $>1.5$ & 0.662 & 79 & 56 & 68 & 68 & 0.023 & $>2.0$ & 0.710 & 83 & 64 & 53 & 88 & 0.0246 \\
\hline
\end{tabular}

AUC: Area under the ROC curve, Sp: specificity, Sn*: sensitivity, PPV: positive predictive value, $N P V$ : negative predictive value, $P$ value: $P>0.05$ non significant... $P<0.05$ : significant $\ldots P<0.001$ : more significant.. $P<0.0001$ : extremely significant.
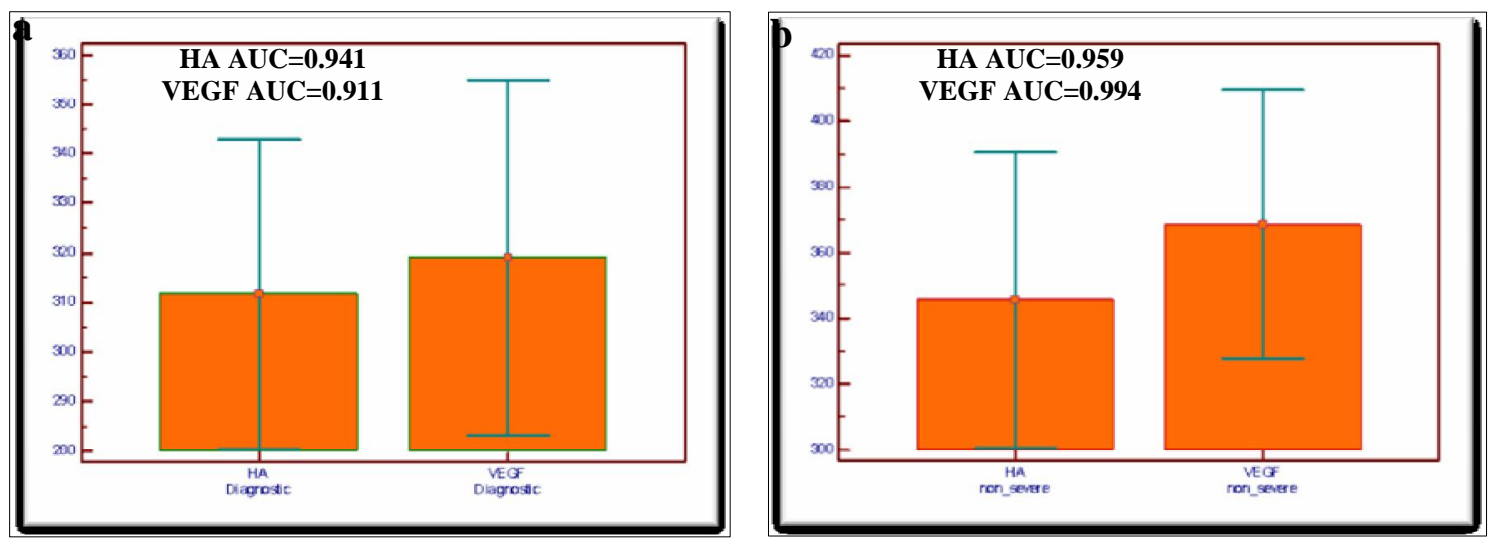

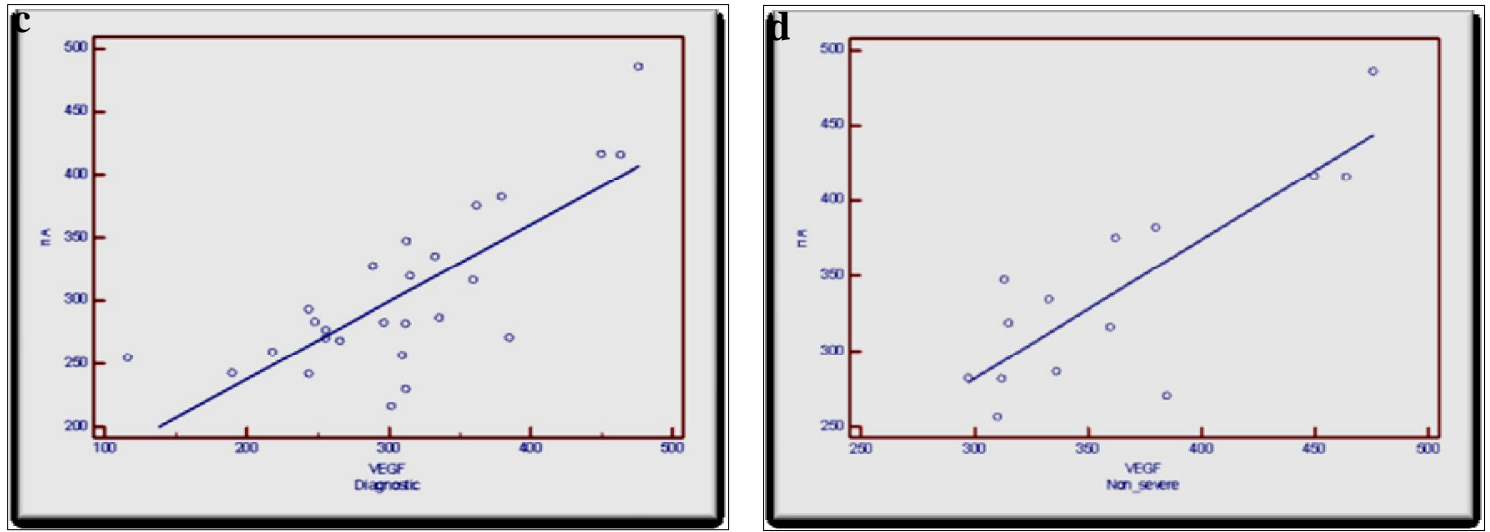

Figure (1) Histogram shown area under curve (AUC) generated by HA and VEGF (a,b) and correlation coefficient between HA and VEGF in all studied groups ( $\mathrm{P}$ value: $\left.<0.0001, \mathrm{r}=0.768^{* *}\right)$ (c) significant fibrosis and in advanced fibrosis $(\mathrm{P}$ value $=$ $\left.0.0003, \mathrm{r}=0.826^{* *}\right)(\mathrm{d})$.

Table (3) Modifications of the diagnostic powers of the selected 8 noninvasive scores after combining the individual results of hyaluronic acid (HA); then vascular endothelial growth factor (VEGF) in discriminating various fibrotic stages.

\begin{tabular}{|c|c|c|c|c|c|c|c|c|c|c|c|c|c|c|}
\hline \multicolumn{15}{|c|}{ Combination with $\mathrm{HA}$} \\
\hline Group & \multicolumn{7}{|c|}{ Non-significantversus signific ant fibrosis } & \multicolumn{7}{|c|}{ Non-severe versus severe fibrosis } \\
\hline Parameter & Cut off & $\mathrm{AUC}$ & $S p$ & $\mathrm{Sn} *$ & $\mathrm{PPV}$ & NPV & $\mathrm{P}$ & Cut off & $\mathrm{AUC}$ & $S p$ & $\mathrm{Sn}^{*}$ & $\mathrm{PPV}$ & NPV & $\mathrm{P}$ \\
\hline AAR & $? 0.91$ & 0.971 & $\begin{array}{c}10 \\
0\end{array}$ & 89 & 100 & 92 & $<0.0001$ & $>1.0$ & 0.989 & 98 & 93 & 93 & 98 & $<0.0001$ \\
\hline GUCI & $>1.0$ & 0.941 & 93 & 85 & 92 & 89 & $<0.0001$ & $>1.56$ & 0.958 & 89 & 93 & 72 & 98 & $<0.0001$ \\
\hline FCI & $>$ & 0.947 & $9 ?$ & 85 & 28.1 & 0.15 & $<0.0001$ & $>1.25$ & 0.957 & 87 & 93 & 68 & 98 & $<0.0001$ \\
\hline FI & $\begin{array}{l}0.095 \\
>3.3\end{array}$ & $0.94 ?$ & 94 & 85 & 92 & 89 & $<0.0001$ & $>3.56$ & 0.961 & 83 & 100 & 63 & 100 & $<0.0001$ \\
\hline $\mathrm{LOK}$ & $>0.5$ & 0.941 & 91 & 85 & 89 & 88 & $<0.0001$ & $>-0.76$ & 0.957 & 93 & 86 & 80 & 96 & $<0.0001$ \\
\hline FIB4 & $>2.67$ & 0.948 & $\begin{array}{c}10 \\
0\end{array}$ & 81 & 100 & 87 & $<0.0001$ & $>3.25$ & 0.966 & 96 & 86 & 86 & 96 & $<0.0001$ \\
\hline KING & $>17.5$ & 0.946 & 85 & 94 & 92 & 89 & $<0.0001$ & $>17.3$ & 0.964 & 89 & 92 & 72 & 98 & $<0.0001$ \\
\hline APRI & $>1.5$ & 0.941 & 94 & 85 & 92 & 87 & $<0.0001$ & $>2.0$ & 0.958 & 87 & 93 & 68 & 98 & $<0.0001$ \\
\hline \multicolumn{15}{|c|}{ Combination with VEGF } \\
\hline $\begin{array}{l}\text { Parameter } \\
\text { AAR }\end{array}$ & $\begin{array}{l}\text { Cut off } \\
? 0.91\end{array}$ & $\begin{array}{r}\text { AUC } \\
0.930\end{array}$ & $\begin{array}{l}S p \\
94\end{array}$ & $\begin{array}{l}S n^{*} \\
81\end{array}$ & $\begin{array}{c}\text { PPV } \\
92\end{array}$ & $\begin{array}{c}\mathrm{NPV} \\
86\end{array}$ & $\begin{array}{c}P \\
<0.0001\end{array}$ & $\begin{array}{c}\text { Cut off } \\
>1.0\end{array}$ & $\begin{array}{l}\text { AUC } \\
0.998\end{array}$ & $\begin{array}{l}\text { Sp } \\
98\end{array}$ & $\begin{array}{l}\mathrm{Sn}^{*} \\
100\end{array}$ & $\begin{array}{c}\text { PPV } \\
93\end{array}$ & $\begin{array}{l}\text { NPV } \\
100\end{array}$ & $\begin{array}{c}P \\
<0.0001\end{array}$ \\
\hline GUCI & $>1.0$ & 0.906 & 76 & 93 & 76 & 93 & $<0.0001$ & $>1.56$ & 0.997 & $9 ?$ & 100 & 93 & 100 & $<0.0001$ \\
\hline FCI & $2>095$ & 0.539 & 33 & 81 & 50 & 69 & 0.6103 & $>1.25$ & 0.995 & 98 & 100 & 93 & 100 & $<0.0001$ \\
\hline FI & $=3.3$ & 0.724 & 94 & 44 & 86 & $6 ?$ & $<0.0001$ & $>3.56$ & 0.994 & 98 & 100 & 93 & 100 & $<0.0001$ \\
\hline $\mathrm{LOK}$ & $=0.5$ & 0.556 & 58 & 59 & 53 & 63 & 0.4675 & $=0.76$ & 0.995 & 97 & 100 & 93 & 100 & $<0.0001$ \\
\hline FIB 4 & $>2.6 ?$ & 0.948 & 58 & 67 & 56 & 67 & 0.1762 & 0.1762 & \multicolumn{6}{|c|}{ No equation } \\
\hline KING & $>17.5$ & 0.802 & 88 & 63 & 81 & 74 & $<0.0001$ & $<0.0001$ & 0.992 & 93 & 100 & 82 & 100 & $<0.0001$ \\
\hline APRI & $>1.5$ & 0.662 & 79 & 56 & 68 & 68 & 0.0236 & 0.0236 & $0.99 ?$ & 98 & 100 & 93 & 100 & $<0.0001$ \\
\hline
\end{tabular}

AUC: Area under the ROC curve, Sp: SPECIFICITY, Sn*: sensitivity, PPV: positive predictive value, NPV: negative predictive value, $P$ value: $P>0.05$ non significant.. $P<0.05$ : significant $\ldots P<0.001$ : more significant... $P<0.0001$ : extremely significant.

Table (4) Comparison of the diagnostic values of HA-Vascular score (HAV score) and hyaluronic acid (HA)+ vascular endothelial growth factor (VEGF) in staging of liver fibrosis.

\begin{tabular}{|c|c|c|c|c|c|c|c|c|c|c|c|c|c|c|}
\hline Group & \multicolumn{7}{|c|}{ Non-significant $¥$ Signific ant } & \multicolumn{7}{|c|}{ Non-severe $¥$ Severe } \\
\hline Parameter & Cut off & $A U C$ & $S p$ & $S n^{4}$ & $P P V$ & $\mathrm{NPV}$ & $P$ & Cut off & $A U C$ & $S p$ & $S n^{*}$ & $P P V$ & $\mathrm{NPV}$ & $P$ \\
\hline HAV score & $>0.583$ & 0.979 & 100 & 89 & 100 & 92 & $<0.0001$ & $>6.27$ & 0.994 & 96 & 100 & 88 & 100 & $<0.0001$ \\
\hline $\mathrm{HA}+\mathrm{VEGF}$ & $>0.26$ & $0.89 ?$ & $9 ?$ & 70 & 95 & 80 & $<0.0001$ & $>-6.39$ & 0.996 & 93 & 100 & 82 & 100 & $<0.0001$ \\
\hline
\end{tabular}



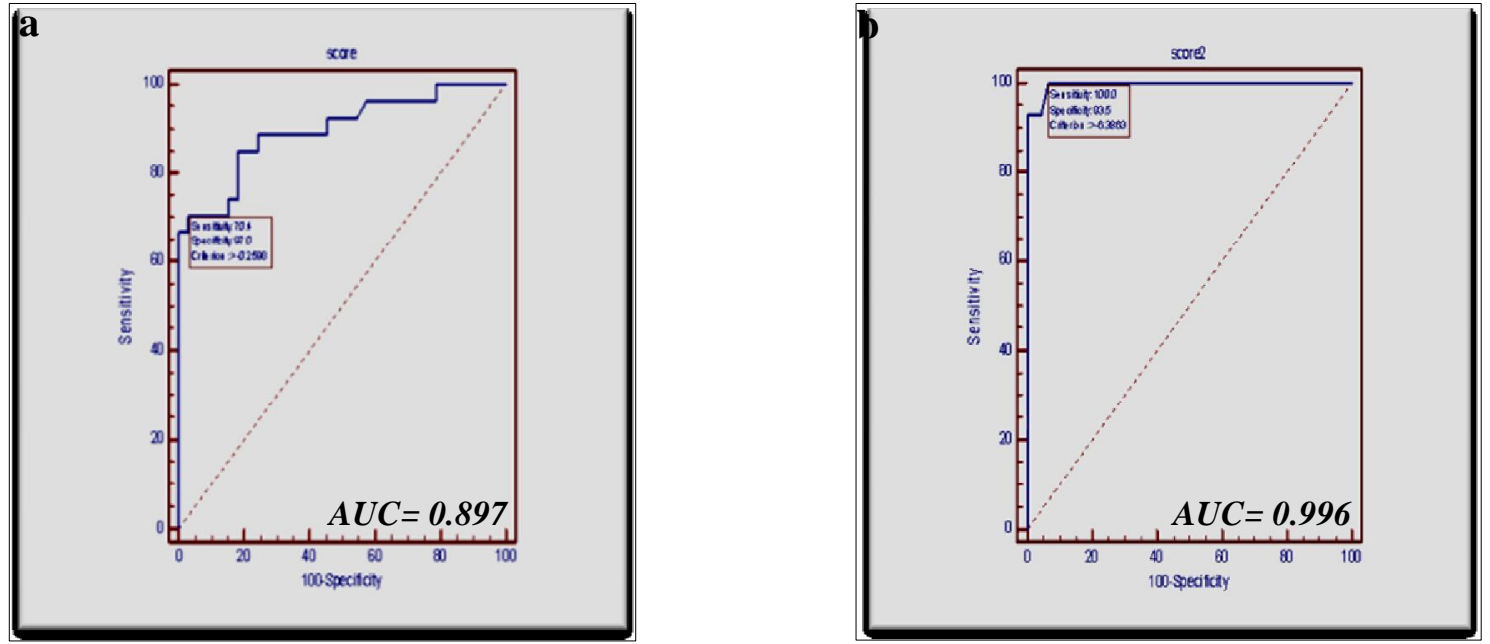

Figure (2) Receiver-operating characteristic curves (ROC) of HA+VEGF for discriminating patients with significant fibrosis; AUC was 0.897 and the best cut-off was at -0.26 (A) and for differentiating patients with severe fibrosis; AUC was 0.996 and the best cut-off was at -6.39 (B).
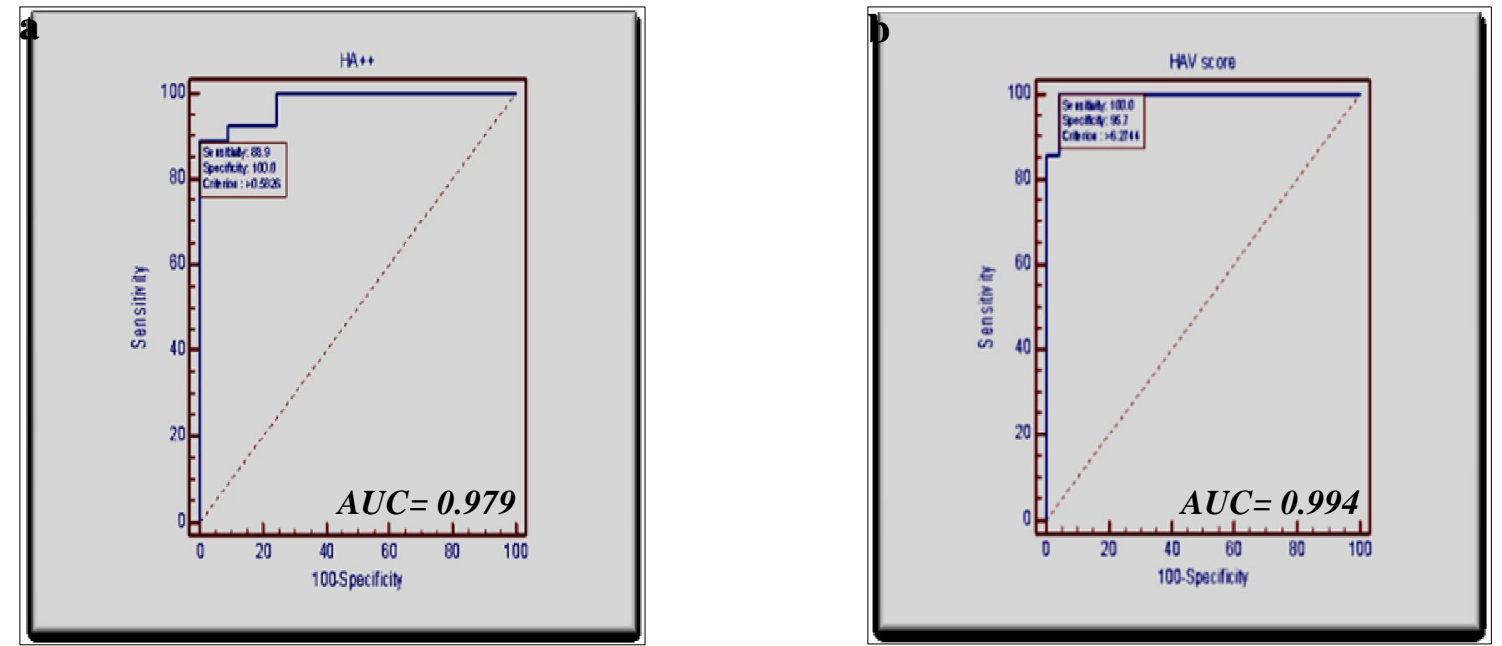

Figure (3) Receiver-operating characteristic curves (ROC) of HAV score on the basis of the HA, VEGF and AAR for differentiating patients with significant fibrosis (F2-F4; AUC $=0.979$ and the best cut-off $=0.583)(\mathrm{A})$ and for differentiating patients with severe fibrosis $(\mathrm{F} 3-\mathrm{F} 4 ; \mathrm{AUC}=0.994$ and the best cut-off $=6.27)(\mathrm{B})$.

Table (5) Diagnostic performances of HAV score and the 5 noninvasive scores for predicting the stages of liver fibrosis in chronic hepatitis $\mathrm{C}$ genotype 4 patients in the validation study.

\begin{tabular}{l|ccccccc}
\hline Parameter & Cut off & AUC & \multicolumn{1}{c}{ Sp } & Sn* & PPV & NPV & P \\
\hline HAV & $>0.583$ & 0.990 & 100 & 92 & 100 & 93 & $<0.0001$ \\
Score & $>0.91$ & 0.701 & 75 & 67 & 71 & 71 & 0.0001 \\
AAR & $>1.0$ & 0.700 & 81 & 54 & 72 & 66 & 0.0001 \\
GUCI & $>3.3$ & 0.740 & 25 & 92 & 53 & 77 & $<0.0001$ \\
FI & $>17.5$ & 0.828 & 83 & 71 & 79 & 75 & $<0.0001$ \\
KING & $>1.5$ & 0.681 & 81 & 54 & 72 & 66 & 0.0007 \\
APRI & & & & & & & \\
\hline
\end{tabular}




\begin{tabular}{|c|c|c|c|c|c|c|c|}
\hline \multicolumn{8}{|c|}{ Advanced fibrosis (F3-F4) } \\
\hline $\begin{array}{l}\text { HAV } \\
\text { score }\end{array}$ & $>-4.4$ & 0.996 & 97 & 100 & 93 & 100 & $<0.0001$ \\
\hline AAR & $>1.0$ & 0.757 & 76 & 73 & 51 & 89 & $<0.0001$ \\
\hline GUCI & $>1.56$ & 0.737 & 84 & 62 & 57 & 86 & 0.0002 \\
\hline FI & $>3.56$ & 0.805 & 92 & 65 & 74 & 88 & $<0.0001$ \\
\hline KING & $>17.3$ & 0.860 & 77 & 85 & 56 & 93 & $<0.0001$ \\
\hline APRI & $>2.0$ & 0.703 & 84 & 62 & 57 & 86 & 0.0032 \\
\hline \multicolumn{8}{|c|}{ Cirrhosis (F4) } \\
\hline $\begin{array}{l}\text { HAV } \\
\text { score }\end{array}$ & $>19.5$ & 0.995 & 98 & 100 & 80 & 100 & $<0.0001$ \\
\hline AAR & $>1.0$ & 0.599 & 82 & 67 & 18 & 98 & 0.7065 \\
\hline GUCI & $>1.1$ & 0.810 & 61 & 100 & 14 & 100 & 0.0043 \\
\hline FI & $>3.6$ & 0.769 & 71 & 100 & 18 & 100 & 0.0001 \\
\hline KING & $>17.5$ & 0.823 & 63 & 100 & 14 & 100 & 0.0019 \\
\hline APRI & $>2.0$ & 0.782 & 88 & 67 & 25 & 98 & 0.0344 \\
\hline
\end{tabular}

AUC: Area under the ROC curve, Sp: SPECIFICITY, Sn*: sensitivity, PPV: positive predictive value, NPV: negative predictive value, $P$ value: $P>0.05$ non significant.. $P<0.05$ : significant $\ldots P<0.001$ : more significant... $P<0.0001$ : extremely significant.

\section{Discussion}

Hepatitis $\mathrm{C}$ virus (HCV) is a heaptotropic virus that has no symptoms in the initial infection. Therefore, most patients do not know about their illness till end stage [28]. Also, this virus can damage the liver and cause accumulation of components of ECM that can either lead to reversible liver fibrosis or irreversible cirrhosis [29]. The latters can distort the hepatic architecture and form capillarization [30]. Therefore, effective HCV screening, early diagnosis and hepatic fibrosis staging are highly relevant for controlling transmission, treating infected patients and, consequently, avoiding end-stage liver disease [31]. Development of liver fibrosis is characterized by the excess deposition of several components of ECM, including various types of collagens, proteoglycans, structural glycoproteins and HA. This excess of organized ECM in the space of Disse (perisinusoidal fibrosis) causes a reduced blood flow through the organ and strangulates the near hepatocytes, influencing the clearance ability of all liver cells [32]. This process extends to distort the hepatic architecture by forming a fibrous scar and developing nodules of regenerating hepatocytes that produces hepatocellular dysfunction and increase the intrahepatic resistance to blood flow [30]. Liver sinusoidal endothelium (LSE) is crucial for normal liver physiology. Cirrhotic livers are characterized by sinusoidal endothelial dysfunction and transformation of the LSE into a continuous, SECs capillarization, i.e. the fenestrae were lost. Then, the disease is progressed and hypoxia was developed [33]. These mechanisms requiring the participation of pro-inflammatory cytokines and growth factors [34]; including VEGF. This is already the case in the present study. This is because the increase in serum HA levels reflect SECs capillarization [35]. Also, the levels of VEGF, which promote and regulate endothelial cell proliferation and migration, matrix remodeling, recruitment of pericytes and neovessels stabilization, were increased with increase in the severity of the disease [36]. A general talk is that, the excessive 
increase in ECM production; including $\mathrm{HA}$, together with the reduction in ECM turnover characterizes liver fibrosis and estimate liver angiogenesis; including VEGF. Angiogenesis is the formation of new vascular structures from preexisting vessels [37] which is essential process for organ growth and repair during and after hepatic disorders [38]. Chronic $\mathrm{HCV}$ infection is one of the main causes for development of hepatic angiogenesis [21]. Generally, the imbalance between hepatic fibrogenesis and angiogenesis can lead to several diseases including malignancy [39]. HA is an essential component of ECM [40] which contributes significantly to cell proliferation and migration, and may also be involved in the progression of some malignant tumors [41]. HA seems to be prognosticator of fibrosis and reflects sinusoidal endothelial cells (SECs) capillarization. This is because it is detected in early stage of liver fibrosis [42]. Also, VEGF is a glycosylated peptide which induces endothelial proliferation and angiogenesis via increasing vascular permeability through disorganization of endothelial junctional proteins which is a highly specific factor for endothelial cells [43] and it is a promoter of fibrogenesis but its actual role in fibrogenesis isn't identified until now. HA act as a promoter of angiogenesis during tissue damage due to its enhancement of the endothelial cell tube formation and its ability to induce the expression of angiogenic factors, such as ICAM-1, VCAM-1 and MMP-2. Therefore, one can suggest that both HA and VEGF act as fibrotic and angiogenic factors [44]. Liver fibrosis is considered a common target of clinical trials in chronic hepatitis $\mathrm{C}(\mathrm{CHC})$ patients. Therefore, its evaluation can yield considerable data and it can be very useful for the diagnosis and prognosis of the disease, and for follow-up of the natural history or progress under therapy. Up till now, the invasive liver biopsy which causes pain, hemorrhage, errors in small samples, can't be repeated and other complications is considered the gold standard test for the evaluation of fibrosis [15]. Therefore, serum HA and VEGF can be used to assess hepatic fibrosis in Egyptian patient's which suffer from chronic $\mathrm{HCV}$ genotype 4 infection. HA was able to differentiate non-significant from those with significant fibrosis with specificity, sensitivity, PPV, NPV and AUC of 94, 85, 92, 89 and 0.941, respectively. The latter parameters were 89, 93, 72, 98 and 0.959 , respectively when the results of HA in severe were compared with those of the non-severe fibrosis. In addition VEGF has specificity, sensitivity, PPV, NPV and AUC of 91, 78, 88, 83 and 0.911 , respectively when the results of VEGF in patients with non-significant fibrosis were compared with those with significant one. The latter parameters of diagnostic power were 93, 100, 82, 100 and 0.994, respectively, when the results of VEGF in patients with severe fibrosis were compared with those with nonsevere fibrosis, tab. (2) and fig. (1), p < $0.0001]$. The elevated diagnostic powers implicate the involvement of HA or VEGF or both in the mechanisms of hepatic fibrogenesis. The latter involvement is not only based on the increase in the deposition of HA in hepatic fibrosis and/or cirrhosis but also on the major role of HA in cell regulation, proliferation and migration. In addition, the growing HA molecule translocates extracellularly through the membrane that increased vascular permeability [45]. Therefore, HA is not just acting as a driving factor of fibrosis but also as a passive player for TGF- $\beta$ to exert its profibrotic effects [40]. Due to the complications of liver biopsy for liver staging in HCV patients, all researchers try to find easy, quick, inexpensive and available scores that should be reproducible. Therefore, HA or VEGF were combined with the numerical values of 8 published non-invasive scores and the parameters of the new diagnostic 
powers were investigated again. Surprisingly, the diagnostic powers of the 8 scores were significantly enhanced after the addition. One example is that the AUC of GUCI was $0.672,0.736$ but became 0.941 and 0.958 after addition of HA and 0.906 and 0.997 after addition of VEGF in discriminating patients with significant from those with non- significant and those with non-severe from those with severe liver fibrosis, respectively, tab. (3). The latter amplify-ications of the diagnostic powers encourage us to test the possible enhancement in the parameters of diagnostic powers or AUCs after addition of the results of HA to those of VEGF. After addition, the AUC was 0.897 for differentiating non significant from those with significant fibrosis and was 0.996 for differentiating non severe from those with severe fibrosis confirming the major role of the direct fibrosis markers in the assessment of hepatic disorders. As before, the role of indirect markers in the assessment of the latter disorders must be kept in our mind. Therefore, the individual results of albumin, ALT, AST or AAR were tested for their abilities to enhance the diagnostic power of the direct markers or not. After testing, AAR was found to add more to the parameters of the diagnostic powers of both HA and VEGF. Thus, the results of AAR were implicated in the construction of the predicting score named HA-Vascular score (HAV score) which was, therefore, consisted of HA, VEGF and AAR. [HAV method $=-35.1+0.14(\mathrm{HA})$ $(\mathrm{ng} / \mathrm{L})+0.03$ (VEGF) $(\mathrm{pg} / \mathrm{ml})+(-6.7)$ (AAR)]. In the estimation study, HAV score could differentiate non-significant fibrosis from those with significant fibrosis with specificity, sensitivity, PPV, NPV and AUC of 100, 89, 100, 92 and 0.979 , respectively. The latter parameters were 96, 100, 88, 100 and 0.994 , respectively when the results of HAV score in severe were compared with those of non-severe fibrosis, tab. (4). In addition, when the results of the diagnostic powers of the latter noninvasive indirect markers were compared with those of HAV score, the statistical results showed that those of HAV score were the best in diagnosing the stages of hepatic fibrosis. Surprisingly, in the validation study HAV score showed high diagnostic performances in differentiating significant, advanced and cirrhosis with specificity, sensitivity, PPV, NPV and AUC of 100,92,100,93 and 0.996; 97, $100,93,100$ and 0.995 and $98,100,80,100$ and 0.995 , respectively, tab. (5). Moreover, the diagnostic powers of other published 8 noninvasive scores, as was originally reported, were lower than those of the candidate HAV score.

\section{Conclusion}

HAV score add more to the reduction of the need of liver biopsy in differentiating liver disorders in the future; especially in cirrhotics, which is actually the aim of the present study.

\section{References}

[1] Abdel-Misih, S., Bloomston, M., (2010). Liver Anatomy. Surgical Clinics of North America; 90 (4): 643653.

[2] Wedro, B., (2015). Liver disease. Medicine net; 1-13.

[3] Buggs, A., (2014). Viral hepatitis. Medscape; 16.

[4] Lavanchy, D., (2011). Evolving epidemiology of hepatitis $\mathrm{C}$ virus. Clin Microbiol Infect; 17: 107-115.

[5] Gupta, E., Bajpai, M., Choudhary, A., (2014). Hepatitis C virus: Screening, diagnosis, and interpretation of laboratory assays, Asian J. Transfus Sci.; 8 (1): 19-25.

[6] Bataller, R., Brenner, D., (2005). Liver fibrosis. The journal of clinical investigation; 115 (2): 209218. 
[7] Griffiths, C., Rooney, C., Brock, A., (2005). Leading causes of death in England and Wales - how should we group causes?. Health Stat $Q$.; (28): 6-17.

[8] Thampanitchawong, P., Piratvisuth, T., (1999). Liver biopsy: complications and risk factors. World J. Gastroenterol.; 5: 301-304.

[9] Wai, C., Greenson, J., Fontana, R., Kalbfleisch, J., Marrero, J., Conjeevaram, H. et al., (2003). A simple noninvasive index can predict both significant fibrosis and cirrhosis in patients with chronic hepatitis C. Hepatology; 38: 518-26.

[10] Afdhal, N., Nunes, D., (2004). Evaluation of liver fibrosis. Am. J. Gastroenterol.; 99: 1160-1174.

[11] Imbert-Bismut, F., Ratziu, V., Pieroni, L., Charlotte, F., Benhamou, Y., Poynard, T., et al., (2001). Biochemical markers of liver fibrosis in patients with hepatitis $\mathrm{C}$ virus infection: a prospective study. Lancet; 357:10691075.

[12] Forns, X., Ampurdanes, S., SanchezTapias, J., Guilera, M., Sans, M., Llovet, J., et al., (2002). Identification of chronic hepatitis $\mathrm{C}$ patients without hepatic fibrosis by a simple predictive model. Hepatology.; 36: 986-992.

[13] Fontana, R., Lok, A., (2002). Noninvasive monitoring of patients with chronic hepatitis C. Hepatology.; 36: S57-S64

[14]. Sebastiani, G., Alberti, A., (2006). Non invasive fibrosis biomarkers reduce but not substitute the need for liver biopsy. World J Gastroenterol; 12 (23): 3682-3694.

[15] Straub, B., Schirmacher, P., (2010). Pathology and biopsy assessment of nonalcoholic fatty liver disease. Dig Dis; 28: 197-202.

[16] Westin, J., Ydreborg, M., Islam, S., Alsiö, A., Dhillon, A., Pawlotsky, J., et al., (2008). A non-invasive fibrosis score predicts treatment outcome in chronic hepatitis $\mathrm{C}$ virus infection. Scand J Gastroenterol. ; 43 (1): 73-80.

[17] Pugh, C., Ratcliffe, P., (2003). Regulation of angiogenesis by hypoxia: role of the HIF system. Nature Medicine; 9: 677-684.

[18] Senger, D., Davis, G., (2011). Angiogenesis. Cold Spring Harb Perspect Biol; 3 (8): a005090.

[19] Thurston, G., (2003). Role of Angiopoietins and Tie receptor tyrosine kinases in angiogenesis and lymphangiogenesis. Cell and Tissue Research; 314 (1): 61-68.

[20] Halfon, P., Bourlière, M., Pénaranda, G., Deydier, R., Renou, C., BottaFridlund, D., et al., (2005). Accuracy of hyaluronic acid level for predicting liver fibrosis stages in patients with hepatitis $\mathrm{C}$ virus. Comparative Hepatology; 4-6.

[21] Salcedo, X., Medina, J., Sanz-Cameno, P., García, L., Samuel, B., Vilchez, M. et al., (2005). The potential of angiogenesis soluble markers in chronic hepatitis C. Hepatology; 42: 696-701.

[22] Tahergorabi, Z., Khazaei, M., (2012). Imbalance of Angiogenesis in Diabetic Complications: The Mechanisms. Int. J of Preventive Medicine; 3 (12): 827-838.

[23] Sterling, R., Lissen, E., Clumeek, N., Sola, R., Correa, M., Montaner, J., et al., (2006). Development of a simple noninvasive index to predict significant fibrosis in patients with HIV/HCV co infection. Hepatology; 43 (6): 1317-1325.

[24] Ahmad, W., Ijaz, B., Javed, F., Gull, S., Kausar, H., Sarwar, M., et al., (2011). A comparison of four fibrosis indexes in chronic HCV: Development of new fibrosis-cirrhosis index (FCI). BMC Gastroenterology; 10: 1186-1471.

[25] Geramizadeh, B., Janfeshan, K., Saberfiroozi, M., (2008). Serum hyaluronic 
acid as a noninvasive marker of hepatic fibrosis in chronic hepatitis B. Saudi Journal of Gastroenterology; 14 (4): 174-177.

[26] Bota, S., Sirli, R., Sporea, I., Focsa, M., Popescu, A., Danila, M., et al., (2011). A new scoring system for prediction of fibrosis in chronic hepatitis C. Hepat Mon.; 11 (7): 548555.

[27] The French METAVIR Cooperative Study Group, (1994). Intraobserver and interobserver variations in liver biopsy interpretation in patients with chronic hepatitis C. Hepatology; 20: 15-20.

[28] Maheshwari, A., Ray, S., Thuluvath, P., (2008). Acute hepatitis C. Lancet; 372 (9635): 321-332.

[29] Montazeri, G., Estakhri, A., Mohamadnejad, M., Nouri, N., Montazeri, F., Mohammad kani, A., et al. (2005). Serum hyaluronate as a noninvasive marker of hepatic fibrosis and inflammation in HBeAg-negative chronic hepatitis B. BMC Gastroenterol.; 5: 32.

[30] Gines, P., Cardenas, A., Arroyo, V., Rodes, J., (2004). Management of cirrhosis and ascites. N. Engl. J. Med.; 350: 1646-1654.

[31] Dehghani, S., Imanieh, M., Haghighat, M., Malekpour, A., Falizkar, Z., (2013). Etiology and complications of liver cirrhosis in children: Report of a single center from Southern Iran. Middle East J Dig Dis.; 5 (1): 41-46.

[32] Ghany, M., Nelson, D., Strader, D., Thomas, D., Seeff, L., (2011). An update on treatment of genotype 1 chronic hepatitis $\mathrm{C}$ virus infection. Practice guideline by the American Association for the Study of Liver Diseases. Hepatology; 54: 1433-1444.

[33] Li, J., Xu, Y., Jiao, H., Wang, W., Mei, Z., Chen, G., (2014). Sumoylation of hypoxia inducible factor- $1 \alpha$ and its significance in cancer. Science China Life Sciences; 57 (7), 657664.

[34] Xu, B., Broome, U., Uzunel, M., Nava, S., Ge, X. et al., (2003). Capillarization of hepatic sinusoid by liver endothelial cell-reactive autoantibodies in patients with cirrhosis and chronic hepatitis. The American Journal of Pathology; 163 (4): 1275-1289.

[35] Semenza, G., (2012). Hypoxia-inducible factors in physiology and medicine. Cell; 148 (3): 399-408.

[36] Sood, G., Slabinski, M., Conrad, S., Luu, L., (2016). Acute liver failure: Medscape; 04.

[37] Tan, B., Messi, W., Laura, M., Akiva, M., Osvaldo, D., (2015). Pericytes at the intersection between tissue regeneration and pathology. Clinical Science; 128 (2): 81-93.

[38] Risau, W., Flamme, I., (1995) Vasculogenesis. Annual Review of Cell and Developmental Biology; 11: 73-91.

[39] Tahergorabi, Z., Khazaei, M., (2012). Imbalance of angiogenesis in diabetic complications: The mechanisms. Int. J. of Preventive Medicine; 3 (12): 827-838.

[40] Liang, X., Zheng, H., (2002). Value of simultaneous determination of serum hyaluronic acid, collagen type IV and the laminin level in diagnosing liver fibrosis. Huang Yi Ke Da Xue Xиe Bao.; 28: 67-68.

[41] Duval, F., Moreno-Cuevas, J., Gonz ález-Garza, M., Maldonado-Bernal, C., Cruz-Vega, D., (2015). Liver fibrosis and mechanisms of the protective action of medicinal plants targeting inflammation and the immune response. Int. J. of Inflammation; 14 p.

[42] Iacovacci, S., Bertolini, L., Manzin, A., Valli, M., Battaglia, M., Ponzetto, A. et al., (1997). Quantitation of hepatitis $\mathrm{C}$ virus RNA production in 
two human bone marrow-derived Bcell lines infected in vitro. Research in Virology; 148: 147-151.

[43] Ferrara, N., (2001). Role of vascular endothelial growth factor in regulation of physiological angiogenesis. American J. of physiology; 280 (6): 1358-1366.

[44] Thomas, S., Vanuystel, J., Gruden, G., Rodríguz, V., Burt, D., Gnudi, L., et al., (2000). Vascular Endothelial Growth Factor Receptors in Human Mesangium in Vitro and in Glomerular Disease. JASN; 11 (7): 1236-1243.

[45] Albeiroti S, Soroosh A, and de la Motte CA. Hyaluronan's Role in Fibrosis: A Pathogenic Factor or a Passive Player? Biomed Res Int. 2015; 2015: 790203. 